\title{
Management of a suspected case of Monkeypox at Vanga Hospital, Kwilu, Republic Democratic of Congo
}

\author{
Article by Mudji e'kitiak \\ M.Sc Clinical Research, Texila American University, Congo \\ E-mail:mudjimedecin@yahoo.fr
}

\section{Introduction}

Monkeypox disease is a zoonosis caused by monkeypox virus, a member of the genus orthopoxvirus, subfamily Chordopoxvirinae, family Poxviridae. It is a virus very similar to the smallpox virus. It is a disease that occurs in tropical Africa, in the Congo Basin. The Congo is the country where you meet the most cases of monkeypox. The disease was first identified in 1958 but the first cases were observed after the eradication of smallpox in 1977, the disease was confused with smallpox for several years (1).

\section{Motivation}

Dermatological diseases are very common in the tropics and children are no exception. A doctor working in an isolated corner is faced with multiple problems including identifying rare diseases which it will be difficult to diagnose and treat.

Monkeypox disease is a zoonosis caused by monkeypox virus, a member of the genus orthopoxvirus, subfamily Chordopoxvirinae, family Poxviridae. It is a virus very similar to the smallpox virus. It is a disease that occurs in tropical Africa, in the Congo Basin.

Monkeypox, a rare disease, occurs with a frequency of two to three cases per year in Evangelist Hospital of Vanga Democratic Republic of Congo. The Congo is the country where you meet the most cases of monkeypox. The disease was first identified in 1958 but the first cases were observed after the eradication of smallpox in 1977, the disease was confused with smallpox for several years (1).

Management is done by both groping especially regarding measures to be taken for the protection of health care, other inpatient and the entire community, knowing that monkeypox is an infectious disease of viral etiology contagious between humans (2). So we reviewed the medical literature about patients with monkeypox and report our experience with a patient admitted with monkeypox in the pediatric ward of the Evangelical Hospital of Vanga.

\section{Patient presentation}

\section{a) Subjectively}

The patient is a 9 years old male that was brought to the hospital pediatric ward for fever and skin rash lasting for about a week.

A week before the start of the fever, the boy used a trap to catch a wild rodent. The local name for this rodent is $M$ wepu. The rodent was noted to have a skin eruption but the boy went ahead and began to roast and it over the fire. His mother upon returning from the field decided that the rodent should not be eaten. One week later, the child presented with fever, dysphagia, asthenia intense physical pain in the mouth, pain in the back and hip. Two days after the onset of fever, parents noticed early macular rash with cervical and submandibular lymphadenopathy. Parents consulted the local health post in Kapimbi in the Vanga health zone. The child visited the Kapimbi health post for two days without improvement so the parents decide to come to the hospital Evangelical vanga for further evaluation. 
This child has seven siblings of which two died of according to the parent of unknown cause. The child has not been fully immunized according to the locally recommended schedule. (Did the child not get any shots or do you know which shots the child did not receive?) This index case was associated with two household contacts also presenting with symptoms suggestive of monkeypox, a fever, rash, and dysphagia and the second child presented with fever, dysphagia but without its eruptions. The sibling with a rash was hospitalized in the same isolation room his sibling. The mother also presented with fever and dysphagia, arthralgia but without skin rash.

Family lives in a town of about 1000 inhabitants. No other children developed similar symptoms in the village. The child attends primary school Kapimbi and no children at that school came down with a similar illness. Mom was afraid of losing her three children especially it has already lost two children in the past.

Community members in the village believe the cause of this family outbreak was a curse because this family has already lost two children. The community leaders did not believe this dangerous disease could be a risk for other members of the community.

\section{b) Objective}

The child weighed $16 \mathrm{~kg}$ to a height of $105 \mathrm{~cm}$ with $\mathrm{P} / \mathrm{T}$ in the middle, with a temperature $38^{\circ} \mathrm{C}$, a heart rate of 100 beats per minute, respiratory rate of 28 cycles per minute. Palpebral and bulbar conjunctiva normal colored and anicteric.

Lymphadenopathy was noted in the posterior cervical sub- mandibular and inguinal regions

Cardiac exam revealed mild tachycardia, normal S1 and S2 with no murmur.

Chest was symmetrical with clear respirations and normal tympani to percussion.

Abdomen was soft, non-tender without organomegaly.

He noted tall umbilicated papules scattered throughout most of the body. Some of these lesions showed secondary infection with honey colored crusting. These lesions were all in similar stage of eruption and showed no surrounding erythema. The rash was most prominent in the trunk, thighs, legs, palms and soles. During hospitalization, the lesions evolved from popular lesions, to vesicle/pustules, then crusts and have finally sloughed.

\section{C) Review of laboratory}

White blood cell count: 16000 élts/mm3

Leukocytes: N 67\%, L 43\%

Blood sedimentation rate: $20 \mathrm{~mm} / \mathrm{H}$

Hemoglobin: 12 gr\%

\section{d) Assessment}

\section{Clinical}

Monkeypox disease with secondary case of a sibling.

\section{E) Management}

\section{Clinic}

We immediately isolated the patient

Have limited medical visits to a doctor and a nurse who wore masks and gloves while providing care.

R / Ampicillin $1 \mathrm{~g} 2$ times a day for 7 day

$\mathrm{R}$ / Paracetamol $500 \mathrm{mg}$ in the case of the mouth

We reassured the child may return to school it once it is healthy because it can infect her friends from school.

We isolated in the same room as the other suspect child and mother, and we reassured and give clear instructions that they should remain in the isolation room until the rash has resolved. 
A team Part of the area is to the village of Akimbo and awareness throughout the population risk of the disease by showing them that it was very important to report any person presenting with fever and rash. Particular emphasis was placed on the community had ideas that it is a curse and this concerned only the family there. They showed that it could happen to anyone because everyone is hunting. Stigma family should end, had told a member of the team area. The central office has worked closely with the hospital on the plan above all monitoring and follow-up.

\section{Review of the literature}

\section{History and character epidemiological}

In Africa, monkeypox has probably existed for several years in sub-Saharan Africa since humans have contracted the virus through contact with infected animals. The reservoir of monkeypox virus is still unknown; however, the data suggest that monkeys, rodents or squirrels or more who live in the forests in Central Africa are the reservoir (3). In our case, the child identified the rodent as a rat locally called Menu. (See picture)

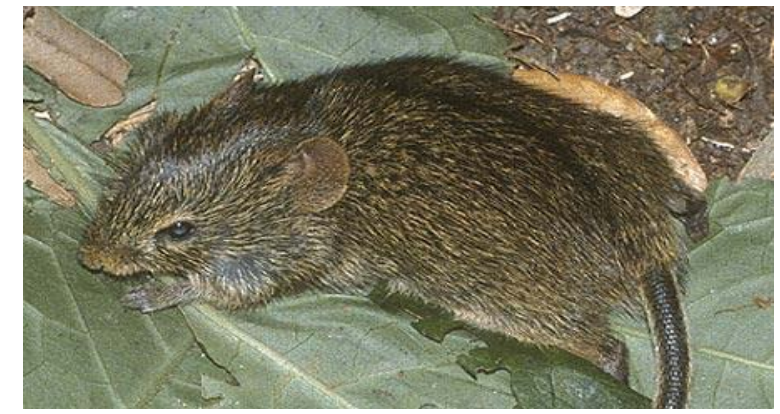

This rat's scientific name is Lemniscomys Rosalia

This is the rat that children identified when we presented with various photos of different rodent species.

Monkeypox was not considered as a separate disease from smallpox until 1970 when, after the elimination of the latter, we continued to have a disease resembling smallpox in rural areas of DR CONGO (4). We are in rural areas where there is most often the case of monkeypox. The virus was first identified in 1958 but the first case was observed in 1970 in Democratic Republic of Congo in a child of 9 months (5-6). Vaccination scale in Central Africa during the global campaign eradication has probably reduced the incidence of monkeypox but the lack of immunity in the generations born since that time and the dependence of the population to feed hunting products explains the resurgence of the disease(4). Early epidemiological studies conducted during the period 1970-1979 detected a total of 47 cases of monkeypox in rainy areas of sub-Saharan Africa, 38 in DR Congo and the rest in Cameroon, Central African Republic, Gabon, Côte d'Ivoire, Liberia and Sierra Leone. (2)

All cases in DR Congo occurred in the rainforests and animal contact was strongly suspected. Secondary transmission has been considered the most likely cause of infection in 4 cases with a secondary attack rate of $7.5 \%$ in the immediate family or member living under the same roof. (7)

Since 1980, the vast majority of cases were reported in DR Congo.

example, to determine whether the monkeypox had the potential to emerge in Central Africa and occupy areas left by smallpox, WHO conducted a monitoring program for the period from 1981 -1986 where 338 of the 404 cases were identified as cases of monkeypox in the period 1970 to 1986 (8).

An animal source was suspected in 245 of 338 cases. Secondary transmission from a human source was assumed in the remaining 93 cases. The majority of cases occurred in children less than 4 years and this distribution suggests waning immunity from small pox vaccination.

Since the end of the monitoring of the WHO, there were some items that were interested in monkeypox. During the period 1986-1992, only 13 items were reported in the literature and none was observed during the period 1993-1996 (9). However, between 1996 and 1997, over 500 cases were 
suspected as cases of monkeypox in Kasai Oriental, but only a small number were laboratory confirmed.

No reports new suspected cases of monkeypox were published until 2001, the year which 31 patients with monkeypox were diagnosed in Equateur province in DRC (4).

Political instability, lack of resources does not allow continuous monitoring of the disease. The disease frequently occurs when cases are not reported.

Between January 1998 and 31 December 2002, a total of 1265 cases were reported to the Ministry of Public Health of the Congo. Samples of 215 cases were analyzed and 88 were due to monkeypox. A monitoring system was installed in Kasai Oriental, with the aim to improve data collection. (4)

In the United States of America, in 2003, cases of monkeypox were reported. 72 cases, 37 were confirmed monkeypox in the laboratory. Prairie dogs or rodents from Africa were suspected source. (10)

\section{Monkeypox Virus}

\section{TRANSMISSION}

The virus is transmitted to humans through the handling of infected animals, as in our case, by direct contact with infected animals or contact with blood, serum or other blood-derived. The transmission is also possible with aerosolized droplets. Human to human transmission is possible but prolonged close contact.

\section{B. Clinical manifestations}

The clinical manifestations of monkeypox are similar to those of smallpox. There is a ten to fourteen day incubation period. The first symptoms appear about two weeks after contact with the virus. The disease begins with fever, malaise, dysphagia, sore mouth, lymph nodes before the rash appears. These macules, papules, pustules and gallbladder, it will form scabs and dander will end (4). Other symptoms may be chills, sweating, headache, back pain and dyspnea. Lymph nodes were observed in approximately $90 \%$ of patients, which is not the case with smallpox and can help in the differential diagnosis are latérocervicales lymphadenopathy, inguinal or submandibular (4). Prodromal stage lasts 1-3 days before appear as rashes. During the first week of the rash, the patient is highly contagious and must be isolated. The diameter of the lesions ranged from 0.5 to $1 \mathrm{~cm}$ and disease progression is almost identical to smallpox. For a period of 2 to 4 weeks, the lesions progress from macules, papules, vesicles, pustules, dry, peel off and become scabs.

Lesions begin to spread to the trunk palm of hands, soles of feet. The lesions may be observed in the mucous membranes of the mouth, tongue and genitals. Other manifestations are soft tissue infection, pneumonia, encephalitis and ocular complication (9).

\section{Diagnosis}

As the clinical manifestations of monkeypox are similar to those of chickenpox and smallpox, accurate diagnosis is crucial for the control of these diseases. A summary table summarizes the situation. 
Table 1. Evaluation criteria for the differential diagnosis of patients with monkeypox, smallpox, and chickenpox.

\begin{tabular}{|c|c|c|c|}
\hline Variable & Monkeypox & Smallpox & Chickenpox \\
\hline Incubation period, days & $7-17$ & $7-17$ & $12-14$ \\
\hline Prodrome period, days & $1-4$ & $2-4$ & $0-2$ \\
\hline \multicolumn{4}{|l|}{ Symptom } \\
\hline Fever, severity & Moderate & Severe & Mild or none \\
\hline Malaise, severity & Moderate & Moderate & Mild \\
\hline Headache, severity & Moderate & Severe & Mild \\
\hline Lymphadenopathy, severity & Moderate & None & None \\
\hline \multicolumn{4}{|l|}{ Lesions } \\
\hline Depth (diameter in mm) & Superficial to deep (4-6) & Deep (4-6) & Superficial (2-4) \\
\hline Distribution & Centrifugal (mainly) & Centrifugal & Centripetal \\
\hline Evaluation & Homogenous rash & Homogenous rash & Heterogeneous rash \\
\hline Time to desquamation, days & $14-21$ & $14-21$ & $6-14$ \\
\hline Frequency of lesions on palms or soles of feet & Common & Common & Rare \\
\hline
\end{tabular}

NOTE. Signs and symptoms of the diseases are not age-specific.

(Site souce of this table)

In the United States, electron microscopy has been used to distinguish between monkeypox and bovine stomatitis.

Thus, although the clinical features are useful in the diagnosis and may help to distinguish the disease, confirmation laboratory is essential for definitive diagnosis. We can try to isolate the virus or use the PCR, ELISA, immunofluorescence test and even histopathological analysis. Unfortunately, many of these methods are relatively nonspecific and are unable to tell the difference between monkeypox virus and other virus's poxvirus. However, immunohistochemically analysis with polyclonal and monoclonal directed against orthopoxviruses, can make the difference between herpes and infections poxvirus. In the past, electron microscopy has played an important role in the diagnosis (11).

Electron microscopy can be a method of choice for the laboratory diagnosis of poxvirus infections, and can provide one of the first clues the cause of a rash illness unknown. This method, however, cannot differentiate between species.

Virus isolation in culture of mammalian cells and characterization techniques of CPR followed by fragment analysis or sequences are considered the definitive means for identifying monkeypox virus (11).

Unlike regions outside Africa where it is essential to distinguish between monkeypox and the deliberate introduction of smallpox, the main diagnostic problem in sub-Saharan Africa is to distinguish monkeypox from chickenpox.

During active disease, laboratory confirmation can be performed by PCR analysis made from liquid vesicles, whereas after the resolution of the disease, serum samples for research IGM antibodies to chickenpox virus can be achieved.

Antipoxivirus The presence of antibodies in unvaccinated individuals with a history of skin rash illness confirms the diagnosis.

\section{Support cases of monkeypox}

For proper treatment, we must define the case.

\section{Definition of case}

Clinical criteria:

Skin rash (macule, papule, vesicle, and pustule) generalized or localized, discrete or congruent. 
Fever greater than $37,4^{\circ} \mathrm{C}$.

Other signs include chills, headache, backache, lymphadenopathy, cough, and dyspnea

Epidemiological criteria:

Exposure to a mammal with signs of illness such as rash, respiratory symptoms, or others.

Exposure to a mammal with or without clinical signs but was in contact with an animal or a human with monkeypox.

Exposure to a probable or confirmed case of suspected monkeypox

Laboratory criteria:

Isolation of monkeypox virus in culture

Presence of virus DNA PCR monkeypox in a clinical specimen.

Demonstration of a morphology resembling a virus an orthopoxvirus by electron microscopy in the absence of exposure to other orthopoxviruses.

Demonstrated presence of an orthopoxvirus in tissue used for the method immunohistochemically test in the absence of exposure to another orthopoxvirus.

\section{Classification of cases}

\section{Suspected case}

Epidemiological criteria skin rash with fever or unexplained two or more symptoms appear in a period less than 21 days

\section{Probable case}

Epidemiological criteria met include fever, rash vésicopustuleuse more skin with onset of symptoms less than 21 days after the last exposure or if rash but not typical elevation IGM antibodies reactive with orthopoxviruses between 7 and 56 days after the early eruptions

\section{Case confirmed}

Those who are included in the test lab

\section{Exclusion criteria}

Another diagnosis can exclude or monkeypox,

The case was reported on the basis of primary or secondary exposure to a wild animal or a human being and who subsequently did not present symptoms or epidemiological or other criteria,

A suspect without developing rashes within ten days following the onset of clinical symptoms consistent with monkeypox.

Cases with undetectable levels of IGM during the period of 7 to 56 days after the rash appear.

INFECTION CONTROL: GENERAL PRECAUTIONS

Anyone who comes to the hospital with fever and rash, a history should look well-made if the person is in contact with the monkey, dog and rat. Once this is proven, measures must be taken to protect health care workers and the public.

Measures to be taken are:

Hygiene hands after contact with an infected patient or environment in which care is provided.

Gowns and Use gloves for contact with the patient.

Protection against the virus by droplets or aerosols. We use the N95 NIOSH certified to filter the air and should be discarded immediately after leaving the room. If the N95 is not available, a surgical mask should be worn to protect against the transmission through contact with droplets. The mask must be worn before entering the patient's room.

Eye protection if splashing through the glasses.

Though managing contaminated waste

Use caution when handling soiled linen to avoid contact with exudates lesions. Dirty laundry should not be shaken or otherwise handled as infectious particles spray. 
Treating the patient avoiding contamination of the skin or clothing. Ensure that the equipment has been cleaned and adjusted accordingly.

\section{Placement of sick}

Patient who presents to the emergency room with fever and rash, maculo papular or vesicular should be immediately placed in isolation as soon as possible. If a negative pressure room is available, it should be used. If this is not available in the next few minutes, to the surgical mask on the patient, covering the patient's skin lesions with clean cloths.

In the hospital, the majority of patients with monkeypox have no need to be hospitalized for medical treatment.

Patients who require hospitalization should be placed in a negative pressure room. If the negative pressure room is not available, a private room should be used.

Vaccination of family caregivers and contacts presumed cases of monkeypox

Vaccination smallpox vaccine is recommended for persons providing care to patients or contacts family of monkeypox. It is better for the caregivers suspected to be vaccinated. The vaccine can be given even before the diagnosis is confirmed if available. Caregivers and family members of suspected cases must comply with the recommendations and precautions to avoid disease.

Monitoring of personal caregiver or other person exposed exposed

Health workers exposed must be followed. Symptoms should be monitored including measurement of body temperature twice a day for 21 days after exposure. Before coming to work each day, the caregiver should be questioned about fever or rash.

Patients who do not require hospitalization for medical indication can be isolated at home. The ability to implement control measures and isolation at home and it varies depends on whether the patient is a child or an adult with monkeypox, if several people in the house are infected, and the number of people who reside in this house, the nature, the extent of damage in each case.

Following principles should be considered and adopted to the extent possible within the family:

1. Isolation at home

People with signs should not leave the house, except as required for follow-up medical care. Should avoid going outside and coming into contact with wild or domestic mammals.

Unexposed persons who do not have a basic need to be at home should not visit the sick. Household members who are not sick should avoid contact with the sick person.

Individuals with extensive lesions that cannot be easily covered with respiratory symptoms should be isolated in a separate room or area member's family whenever possible.

2. The person with a sign must wear a surgical mask in particular that this respiratory signs. If this is not possible, other members of the household should consider wearing a surgical mask in the presence of the person with symptoms.

3. Skin lesions should be covered to the extent possible to minimize the risk of contact with others.

4. Disposable gloves should be worn for direct contact with oozing lesions and discard after use.

5. The hand hygiene (washing hands with soap or other disinfectant) must be performed by person's infected household contacts after touching contaminated areas of the body, clothing or environment that may have contact with infectious lesions.

6. Bedding, towels, clothes can be washed in a standard washing machine with warm water and detergent, bleach can be added. Precautions should be used when handling soiled linen and avoid contact with other materials. The cloths must not be shaken or otherwise manipulate to spray the infectious particles.

7. Dishes and other utensils should not be shared, but the segregation of specific utensils for use by an infected person is not necessary. Dirty dishes and utensils should be washed in a dishwasher or by hand with warm water and soap

8. Contaminated surfaces should be cleaned and disinfected. Disinfectants can be used. 
9. Contaminated materials must be bagged and placed in a container for disposal with household garbage.

\section{Period of isolation}

decisions relating to discontinuation of isolation precautions should be taken after consultation with all services health.

For people with a rash vésiculopustuleuse, isolation measures should be continued until the lesions become scabs.

Precautionary shutdown after isolation, affected individuals should avoid close contact with immunocompromised people until all lesions that disappear. This applies to the case of HIV, diabetes, cancer, emphysema.

For people who develop symptoms such as fever, sore throat, cough without rash, isolation precautions should be continued for 7 days after the onset of fever. If the rash does not develop during this time, isolation measures may be interrupted. Those affected must continue to monitor symptoms for 14 days. If symptoms recur or if a rash develops, the health department must be notified.

Affected individuals should not donate blood, cells, tissues, organs, breast milk, semen or while they are sick or during monitoring.

Contacts asymptomatic animals or humans suspected of having monkeypox should be placed under surveillance for 21 days symptoms bitter their latest exhibitions. It monitors fever, sore throat, cough, and rash. Closer monitoring is desirable.

Asymptomatic contacts should continue their daily activities (go to school, work) but should stay close to home throughout the duration of the surveillance. However it may be prudent to exclude preschoolers to gardiennerie or other group.

\section{Conclusion}

Monkeypox is a viral disease although rare but present in our area. Two to three cases are reported each year in the hospital Vanga. No protocol or concrete plan of care does not exist in our area.

Recognition of the disease by thorough anamnesis, physical examination and thorough epidemiological data are tools for health personnel.

Rats in our case are clearly defined as a reservoir of the disease from clear and reliable evidence of the family.

Once diagnosed, the measures detailed above should be switched to the management of the disease.

\section{References}

[1]. Aysegul N, Anne W, Sina B, Chris A. Reemergence of Monkeypox: Prevalence, Diagnostics, and Countermeasures. Clin Infect Dis. 2005 Dec 15;41(12):1765-71. Epub 2005 Nov 11

[2]. Foster SO, Brink EW, Hutchins DL, et al. Human monkeypox. Bull World Health Organization 1972;46:569-76.

[3]. Gentile M, Gelderblom HR. Rapid viral diagnosis: role of electron microscopy. New Microbiol 2005; 28:112.

[4]. Heymann DL, Szczeniowski M, Esteves K. Re-emergence of monkeypox in Africa: a review of the past six years. Br Med Bull 1998; 54:693-702

[5]. Jezek Z, Arita I, Mutombo M, Dunn C, Nakano JH, M. Szczeniowski Four generation of probable person to person transmission of human monkeypox. Am j Epidemiol 1986; 123:1004-12

[6]. Jezek Z, Fenner F. Human monkeypox. In: Melnick JL, ed. Monographs in virology ol. 17. Basel, Switzerland: Karger,1 988

[7]. Khodakevich L, Jezek Z, Messinger D. Monkeypox virus: ecology and public health significance. Bull World Health Organ 1988; 66:747-52.

[8]. Ladnyj ID, Ziegler P, Kima E. A human infection caused by monkeypox virus in Basankusu Territory, Democratic Republic of the Congo. Bull World Health Organ 1972;46:593-7. 
Texila International Journal of Medicine

Volume 4, Issue 2, Dec 2016

[9]. Multistate outbreak of monkeypox-Illinois, Indiana, and Wisconsin, 2003. MMWR Morb Mortal Wkly Rep 2003; 52:537-40.

[10]. The global eradication of smallpox: final report of the global commission for the certification of smallpox eradication. Geneva, Switzerland: World Health Organization, 1980.

[11]. Inger K. Damon. Status of human monkeypox: clinical disease, epidemiology and research. Vaccine. Dec. 30 2011, 29 Suppl 4: D54-9. Epub 2011 Dec 18. 